\title{
Teaching NeuroImages: Rare skull base involvement in neurosarcoidosis
}

Christoph Friedrich Mahler, MD, Ashok Adams, MD, Krishna Suchak, MD, and

Sharmilee Gnanapavan, MD, PhD

Neurology ${ }^{\circledR}$ 2020;95:e939-e940. doi:10.1212/WNL.0000000000010082

Figure 1 Imaging

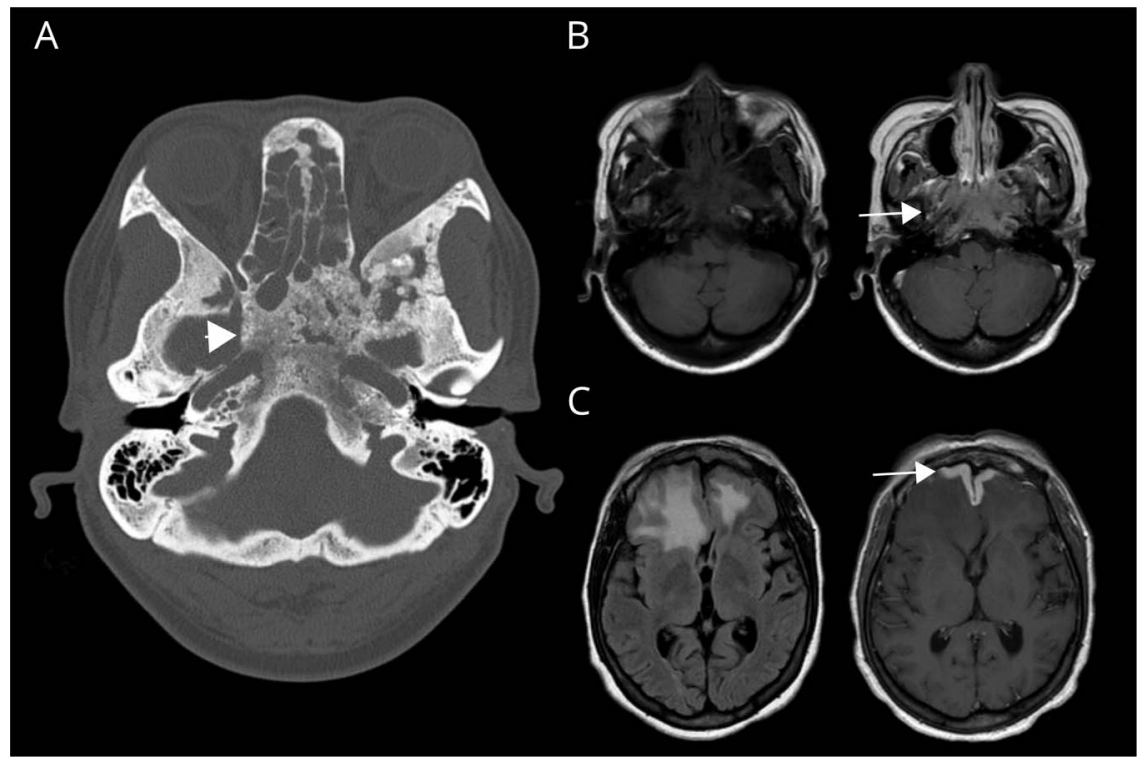

(A) Sclerotic and potentially ground-glass appearance of skull base (arrowhead) (CT). (B) Contrast enhancement of the irregular central skull base changes (arrow); left: MRI T1; right: MRI T1 with gadolinium contrast. (C) White matter edema and gadolinium-enhancing frontal pachymeningeal thickening (arrow); left: MRI FLAIR; right: MRI T1 with gadolinium contrast.

A 57-year-old woman was admitted with a tonic-clonic seizure on a background of systemic sarcoidosis with uveitis and hilar lymphadenopathy. She had previously been well and stopped steroids 1 year before presentation. CT showed a sclerotic lesion with focal lucent areas in the skull base (figure 1, A and B). MRI revealed a frontal fluid-attenuated inversion recovery hyperintense edematous lesion with meningeal enhancement in gadolinium T1 (figure 1C). This appearance on imaging evokes broad differential diagnoses such as sarcoidosis, fungal (although she had increasing lesion size despite 6-month treatment with amphotericin B), craniofacial fibrous dysplasia, atypical lymphoma, nasopharyngeal carcinoma, myeloma, and tuberculosis, therefore necessitating biopsy for definitive evaluation. Transsphenoidal biopsy revealed noncaseating granulomatous inflammation and the patient was started on oral glucocorticoid therapy for sarcoidosis (figure 2). Neurosarcoidosis with skull base bone involvement is uncommonly reported. ${ }^{1,2}$

\section{Study funding}

No targeted funding reported.

\section{Correspondence}

Dr. Gnanapavan

sharmilee.gnanapavan@ nhs.net
MORE ONLINE

$\rightarrow$ Teaching slides

links.lww.com/WNL/

B140 

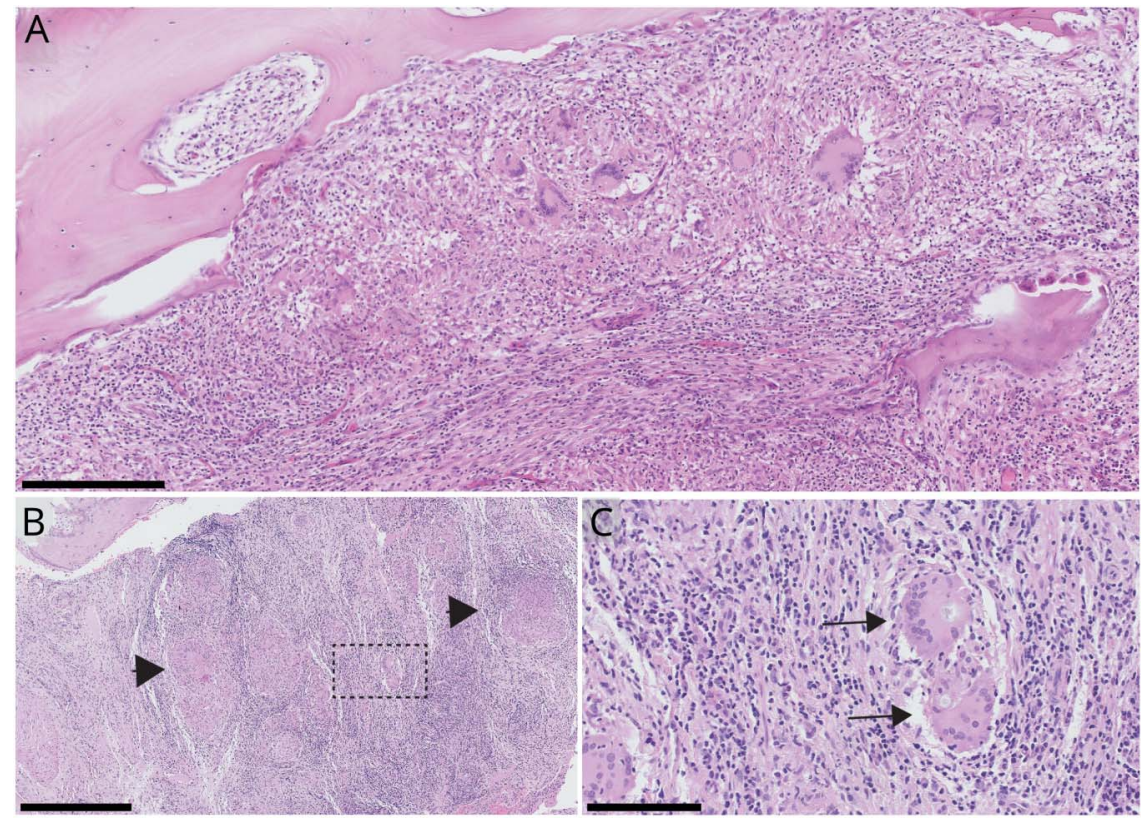

Transsphenoidal biopsy. (A) Numerous granulomata are seen between the vital woven bony trabeculae of the sphenoid wall. (B) Noncaseating granulomas (arrowheads). (C) Multinucleate giant cells (arrows). Hematoxylin \& eosin; scale: (A) $250 \mu \mathrm{m}$; (B) $500 \mu \mathrm{m}$; (C) $100 \mu \mathrm{m}$

\section{Disclosure}

The authors report no disclosures relevant to the manuscript. Go to Neurology.org/ $\mathrm{N}$ for full disclosures.

Appendix Authors

\begin{tabular}{lll}
\hline Name & Location & Contribution \\
\hline $\begin{array}{l}\text { Christoph } \\
\begin{array}{l}\text { Friedrich } \\
\text { Mahler, MD }\end{array}\end{array}$ & $\begin{array}{l}\text { Royal } \\
\text { London } \\
\text { Hospital, UK }\end{array}$ & $\begin{array}{l}\text { Data collection, drafting and revising } \\
\text { the manuscript }\end{array}$ \\
\hline $\begin{array}{l}\text { Ashok Adams, } \\
\text { MD }\end{array}$ & $\begin{array}{l}\text { Royal } \\
\text { London }\end{array}$ & $\begin{array}{l}\text { Radiologic imaging analysis and } \\
\text { report }\end{array}$ \\
& Hospital, UK & \\
\hline
\end{tabular}

Appendix (continued)

\begin{tabular}{lll}
\hline Name & Location & Contribution \\
\hline $\begin{array}{l}\text { Krishna Suchak, } \\
\text { MD }\end{array}$ & $\begin{array}{l}\text { Royal } \\
\text { London } \\
\text { Hospital, UK }\end{array}$ & $\begin{array}{l}\text { Histology microscopy, analysis and } \\
\text { report }\end{array}$ \\
\hline $\begin{array}{l}\text { Sharmilee } \\
\text { Gnanapavan, } \\
\text { MD, PhD }\end{array}$ & $\begin{array}{l}\text { Royal } \\
\text { London } \\
\text { Hospital, UK }\end{array}$ & $\begin{array}{l}\text { Study supervision, clinical care for } \\
\text { the patient and critical comments } \\
\text { during manuscript revision }\end{array}$ \\
\hline
\end{tabular}

\section{References}

1. Teirstein AS, Wolf BS, Siltzbach LE. Sarcoidosis of the skull. N Engl J Med 1961;265: 65-68.

2. Carlson ML, White JR, Espahbodi M, et al. Cranial base manifestations of neurosarcoidosis: a review of 305 patients. Otol Neurotol 2015;36:156-166. 


\section{Neurology}

\section{Teaching NeuroImages: Rare skull base involvement in neurosarcoidosis Christoph Friedrich Mahler, Ashok Adams, Krishna Suchak, et al. \\ Neurology 2020;95;e939-e940 Published Online before print June 30, 2020 \\ DOI 10.1212/WNL.0000000000010082}

This information is current as of June 30, 2020

$\begin{array}{ll}\begin{array}{l}\text { Updated Information \& } \\ \text { Services }\end{array} & \begin{array}{l}\text { including high resolution figures, can be found at: } \\ \text { http://n.neurology.org/content/95/7/e939.full }\end{array} \\ \text { References } & \text { This article cites } 2 \text { articles, } 0 \text { of which you can access for free at: } \\ \text { http://n.neurology.org/content/95/7/e939.full\#ref-list-1 } & \\ \text { This article, along with others on similar topics, appears in the } \\ \text { following collection(s): } \\ \text { All Immunology } \\ \text { http://n.neurology.org/cgi/collection/all_immunology } \\ \text { CT } \\ \text { http://n.neurology.org/cgi/collection/ct } \\ \text { MRI } \\ \text { http://n.neurology.org/cgi/collection/mri } \\ \text { Information about reproducing this article in parts (figures,tables) or in } \\ \text { its entirety can be found online at: } \\ \text { http://www.neurology.org/about/about_the_journal\#permissions } \\ \text { Information about ordering reprints can be found online: } \\ \text { Pttp://n.neurology.org/subscribers/advertise }\end{array}$

Neurology ${ }^{\circledR}$ is the official journal of the American Academy of Neurology. Published continuously since 1951, it is now a weekly with 48 issues per year. Copyright (C 2020 American Academy of Neurology. All rights reserved. Print ISSN: 0028-3878. Online ISSN: 1526-632X.

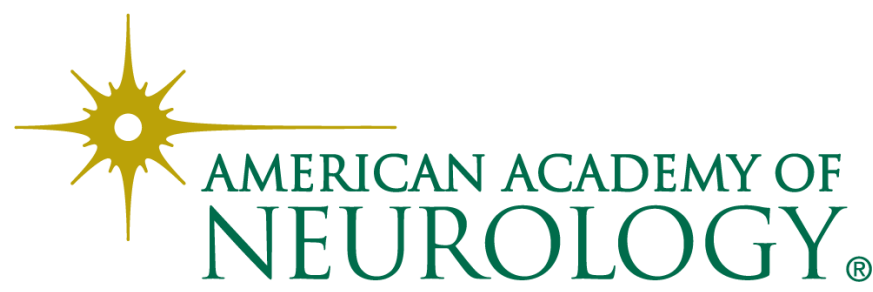

